\title{
Coupled-oscillator model for nonlinear optical activity
}

\author{
M. A. Belkin, Y. R. Shen \\ Department of Physics, University of California, Berkeley, CA 94720 and Materials Sciences \\ Division, Lawrence Berkeley National Laboratory, Berkeley, CA 94720 \\ (Fax: +1-510-643-8923,E-mail: shenyr@socrates.berkeley.edu) \\ C. Flytzanis \\ Laboratoire de Physique de la Matière Condensée, Ecole Normale Supérieure, 24 rue Lhomond, \\ 75231 Paris Cedex 05, France
}

\begin{abstract}
The coupled-oscillator model, previously used to describe linear optical activity, is extended to the study of nonlinear optical activity of dimer-like chiral molecules. The result shows good agreement between theory and experiment for optically active sum-frequency generation from 1,1'-bi-2-naphthol solution. It is seen that linear and nonlinear optical activities generally probe different aspects of the chiral structure of the molecules.
\end{abstract}

Recently nonlinear optical techniques such as second harmonic generation (SHG) and sum-frequency generation (SFG) have been demonstrated to be potentially useful for probing molecular chirality in various circumstances [1-4]. Contrary to the conventional optical techniques such as linear optical birefringence, circular dichroism, and Raman optical activity, SHG and SFG are electric-dipole allowed in a chiral medium so that they have the sensitivity to detect chiral response even from a surface monolayer. This opens many research possibilities in various disciplines of chemistry and biology. 
To establish SHG and SFG as useful tools for studying molecular chirality, we need to relate the chiral nonlinear optical response from molecules to the chiral molecular structure. We would also like to see whether the structural information thus deduced is different from that obtained by other optical techniques. For this purpose, we must search for a microscopic understanding of chirality at molecular level. Theoretical works on nonlinear optical activity of chiral molecules are rare [6-8] and most of them are limited to $a b$ initio calculations. They fail to provide a clear physical picture relating the results to the molecular chiral structure. It would be helpful if one could find simple models to describe chiral responses of molecules even though they may be less accurate. Such models do exist for interpretation of linear optical activity of selected types of molecules [9]. We believe that the same models can also be used to describe their nonlinear optical activity. In a recent article, Hache et al discussed two classical models to describe molecular chiral response in SHG, but the work was only meant to be illustrative.

In this paper, we consider the quantum coupled-oscillator model that is applicable to chiral molecules composed of two coupled monomers in a twisted geometry (with a $C_{2}$ symmetry) [10]. We use 1,1'-bi-2-naphthol (BN) molecules as an example, with which the calculation can be compared with experiment. We focus on SFG in transmission near electronic resonances of $\mathrm{BN}$ in a solution. The model predictions are in good agreement with the experimental results $[4,5]$.

SFG from a medium results from a nonlinear polarization:

$$
\vec{P}^{(2)}\left(\omega=\omega_{1}+\omega_{2}\right)=\epsilon_{0} \overleftrightarrow{\chi}^{(2)}\left(\omega=\omega_{1}+\omega_{2}\right): \vec{E}_{1}\left(\omega_{1}\right) \vec{E}_{2}\left(\omega_{2}\right)
$$

induced in the medium by input fields $\vec{E}_{1}$ and $\vec{E}_{2}$ at frequencies $\omega_{1}$ and $\omega_{2}$, respectively [11]. SHG is a special case of SFG with $\omega_{1}=\omega_{2}$. The nonlinear susceptibility $\overleftrightarrow{\chi}^{(2)}$ is related to the nonlinear polarizability $\overleftrightarrow{\alpha}^{(2)}$ of the molecules in the medium by the expression:

$$
\overleftrightarrow{\chi}^{(2)}=\frac{1}{\epsilon_{0}} N L(\omega) L\left(\omega_{1}\right) L\left(\omega_{2}\right)<\overleftrightarrow{\alpha}^{(2)}>
$$

where $N$ is the molecular density, $L\left(\omega_{i}\right)$ is the Lorentz local-field correction factor with $\epsilon\left(\omega_{i}\right)$ 
being the dielectric constant at $\omega_{i}$, and the angular brackets denote an average over the molecular orientations.

The quantum-mechanical expression for $\overleftrightarrow{\alpha}^{(2)}$ takes the form [12]:

$$
\begin{aligned}
\alpha_{i j k}^{(2)}= & \frac{1}{\hbar^{2}} \sum_{n, n^{\prime}} \frac{\left(\mu_{i}\right)_{g n}\left(\mu_{j}\right)_{n n^{\prime}}\left(\mu_{k}\right)_{n^{\prime} g}}{\left(\omega-\omega_{n g}+i \Gamma_{n g}\right)\left(\omega_{2}-\omega_{n^{\prime} g}+i \Gamma_{n^{\prime} g}\right)}+\frac{\left(\mu_{i}\right)_{g n}\left(\mu_{j}\right)_{n^{\prime} g}\left(\mu_{k}\right)_{n n^{\prime}}}{\left(\omega-\omega_{n g}+i \Gamma_{n g}\right)\left(\omega_{1}-\omega_{n^{\prime} g}+i \Gamma_{n^{\prime} g}\right)}+ \\
& +\frac{\left(\mu_{i}\right)_{n g}\left(\mu_{j}\right)_{n^{\prime} n}\left(\mu_{k}\right)_{g n^{\prime}}}{\left(\omega+\omega_{n g}+i \Gamma_{n g}\right)\left(\omega_{2}+\omega_{n^{\prime} g}+i \Gamma_{n^{\prime} g}\right)}+\frac{\left(\mu_{i}\right)_{n g}\left(\mu_{j}\right)_{g n^{\prime}}\left(\mu_{k}\right)_{n^{\prime} n}}{\left(\omega+\omega_{n g}+i \Gamma_{n g}\right)\left(\omega_{1}+\omega_{n^{\prime} g}+i \Gamma_{n^{\prime} g}\right)}- \\
& -\frac{1}{\left(\omega-\omega_{n^{\prime} n}\left(\mu_{j}\right)_{n g}\left(\mu_{k}\right)_{g n^{\prime}}+i \Gamma_{n n^{\prime}}\right)} \cdot\left(\frac{1}{\omega_{2}+\omega_{n^{\prime} g}+i \Gamma_{n^{\prime} g}}+\frac{1}{\omega_{1}-\omega_{n g}+i \Gamma_{n g}}\right)- \\
& -\frac{\left(\mu_{i}\right)_{n^{\prime} n}\left(\mu_{j}\right)_{g n^{\prime}}\left(\mu_{k}\right)_{n g}}{\left(\omega-\omega_{n n^{\prime}}+i \Gamma_{n n^{\prime}}\right)} \cdot\left(\frac{1}{\omega_{2}-\omega_{n g}+i \Gamma_{n g}}+\frac{1}{\omega_{1}+\omega_{n^{\prime} g}+i \Gamma_{n^{\prime} g}}\right)
\end{aligned}
$$

where $\hat{i}, \hat{j}, \hat{k}$ refer to the molecular coordinates, $n$ and $n^{\prime}$ denote the eigenstates, $\omega_{a b}$ and $\Gamma_{a b}$ are the transition frequency and the damping factor for the transition from state $\mid a>$ to state $\mid b>$ and we assume that all molecules are initially in ground state $\mid g>$.

We now consider a dimer molecule consisting of two identical monomers. The BN molecule, shown in Fig. 1a, is an example. In the coupled-oscillator model, the ground electronic state of the dimer is given by:

$$
|g>=| 0>\mid 0^{\prime}>
$$

where $\mid 0>$ and $\mid 0^{\prime}>$ refer to the ground electronic states of the monomers. The excited electronic states are taken as the symmetrized and antisymmetrized functions of the products of the individual states of the two monomers. For example, the first set of excited states of the dimer has the expressions:

$$
\mid e_{1_{ \pm}}>=\frac{1}{\sqrt{2}}\left(|0>| 1^{\prime}> \pm|1>| 0^{\prime}>\right)
$$

with $\mid 1>$ and $\mid 1^{\prime}>$ denoting the first excited states of the two individual monomers. The degeneracy of $\mid e_{1_{ \pm}}>$is lifted by coupling between the two monomers. The splitting, known as the Davydov exciton splitting, depends on the coupling strength so that:

$$
E_{e_{1_{+}}}-E_{e_{1_{-}}}=2<1\left|<0^{\prime}\right| V|0>| 1^{\prime}>
$$


where $E_{e_{1_{+}}}$and $E_{e_{1_{-}}}$are the energies of states $\mid e_{1_{+}}>$and $\left|e_{1_{-}}\right\rangle$, and $V$ is the interaction Hamiltonian that describes the dipole-dipole interaction of the monomers. If $<1\left|<0^{\prime}\right| V|0>| 1^{\prime}>$ is positive, $\mid e_{1_{+}}>$lies higher than $\mid e_{1_{-}}>$.

The electric-dipole operator for a dimer in Eq. (3) can be approximated as the sum of the electric-dipole operators of the monomers:

$$
\vec{\mu}_{D}=\vec{\mu}+\vec{\mu}^{\prime}
$$

We can then substitute the approximate dimer states of Eqs. (4) and (5) into Eq. (3) to find $\overleftrightarrow{\alpha}^{(2)}$. For later comparison with experiment, we consider the case where the sum frequency $\omega$ is near resonance with the lowest electronic transitions $\omega_{e_{1_{ \pm}} g}$ while $\omega_{1}$ and $\omega_{2}$ are off resonance. Keeping only the near resonant terms in Eq. (3) we find:

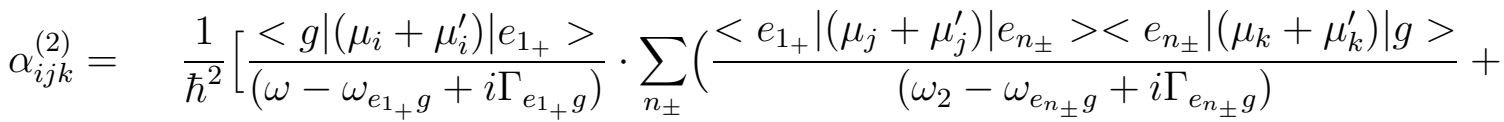

$$
\begin{aligned}
& \left.+\frac{<e_{1_{+}}\left|\left(\mu_{k}+\mu_{k}^{\prime}\right)\right| e_{n_{ \pm}}><e_{n_{ \pm}}\left|\left(\mu_{j}+\mu_{j}^{\prime}\right)\right| g>}{\left(\omega_{1}-\omega_{e_{n_{ \pm}}}+i \Gamma_{e_{n_{ \pm}}}\right)}\right)- \\
& -\frac{<g\left|\left(\mu_{i}+\mu_{i}^{\prime}\right)\right| e_{1_{-}}>}{\left(\omega-\omega_{e_{1} g}+i \Gamma_{e_{1} g}\right)} \cdot \sum_{n_{ \pm}}\left(\frac{<e_{1_{-}}\left|\left(\mu_{j}+\mu_{j}^{\prime}\right)\right| e_{n_{ \pm}}><e_{n_{ \pm}}\left|\left(\mu_{k}+\mu_{k}^{\prime}\right)\right| g>}{\left(\omega_{2}-\omega_{e_{n_{ \pm}}}+i \Gamma_{e_{n_{ \pm}} g}\right)}+\right. \\
& \left.\left.+\frac{<e_{1_{-}}\left|\left(\mu_{k}+\mu_{k}^{\prime}\right)\right| e_{n_{ \pm}}><e_{n_{ \pm}}\left|\left(\mu_{j}+\mu_{j}^{\prime}\right)\right| g>}{\left(\omega_{1}-\omega_{e_{n_{ \pm}}}+i \Gamma_{e_{n_{ \pm}} g}\right)}\right)\right]
\end{aligned}
$$

where the summation is over excited states $\mid e_{n_{ \pm}}>$defined by:

$$
\mid e_{n_{ \pm}}>=\frac{1}{\sqrt{2}}\left(|0>| n^{\prime}> \pm|n>| 0^{\prime}>\right)
$$

Note that other excited states of the forms $|i>| i^{\prime}>$ and $\frac{1}{\sqrt{2}}\left(|i>| j^{\prime}> \pm|j>| i^{\prime}>\right)$ with $i \neq j \neq 0$ do not come in because they cannot be connected to the ground state $\mid g>$ by $\vec{\mu}_{D}$. The above expression for $\overleftrightarrow{\alpha}^{(2)}$ depends on dipole moments of the monomers and their relative orientation in a molecule. The chiral elements of $\overleftrightarrow{\alpha}^{(2)}$ can be nonvanishing because the monomers are chiral, but if the dimer is composed of two achiral monomers, then it is the relative orientation of the two that makes the dimer molecule chiral.

Inserting Eq. (8) into Eq. (2) and adopting a proper orientational distribution, one can obtain $\overleftrightarrow{\chi}^{(2)}$ for a surface or bulk with known structure. Here, we focus on $\overleftrightarrow{\chi}^{(2)}$ for bulk liquids 
in which the molecules are isotropically oriented. Under the electric-dipole approximation, $\overleftrightarrow{\chi}^{(2)} \neq 0$ only if the liquid is chiral and the only nonvanishing elements of $\overleftrightarrow{\chi}^{(2)}$ are $\chi_{i j k}^{(2)}$ with $i \neq j \neq k$ [13]. From isotropic symmetry, we have, following Eq. (2):

$$
\begin{aligned}
\chi_{\text {chiral }} & =\frac{1}{\epsilon_{0}} N L(\omega) L\left(\omega_{1}\right) L\left(\omega_{2}\right) \alpha_{\text {chiral }} \\
\alpha_{\text {chiral }} & \equiv<\alpha_{\xi \eta \zeta}^{(2)}(\hat{i} \cdot \hat{\xi})(\hat{j} \cdot \hat{\eta})(\hat{k} \cdot \hat{\zeta})>=\frac{1}{6}\left(\alpha_{\xi \eta \zeta}^{(2)} \cdot e_{\xi \eta \zeta}\right)
\end{aligned}
$$

Here, local field corrections $L\left(\omega_{i}\right)=\left[\epsilon\left(\omega_{i}\right)+2\right] / 3, \xi, \eta, \zeta$ refer to the molecular coordinates, $e_{i j k}$ is the Levi-Civita tensor and summation over the repeated indices is implied. Note that $\chi_{\text {chiral }}$ or $\alpha_{\text {chiral }}$ changes sign upon inversion and therefore should vanish for achiral liquids or racemic mixtures. Then from Eq. (8), we can show:

$$
\begin{aligned}
\alpha_{\text {chiral }} & =\frac{1}{\hbar^{2}} \cdot\left(\omega_{1}-\omega_{2}\right) \cdot \\
& \cdot\left[\frac{<g\left|\left(\vec{\mu}+\vec{\mu}^{\prime}\right)\right| e_{1_{+}}>}{\left(\omega-\omega_{e_{1_{+}} g}+i \Gamma_{e_{1_{+}}}\right)} \cdot \sum_{n_{ \pm}} \frac{<e_{1_{+}}\left|\left(\vec{\mu}+\vec{\mu}^{\prime}\right)\right| e_{n_{ \pm}}>\times<e_{n_{ \pm}}\left|\left(\vec{\mu}+\vec{\mu}^{\prime}\right)\right| g>}{\left(\omega_{2}-\omega_{e_{n_{ \pm}}}\right)\left(\omega_{1}-\omega_{e_{n_{ \pm}} g}\right)}+\right. \\
& \left.+\frac{<g\left|\left(\vec{\mu}+\vec{\mu}^{\prime}\right)\right| e_{1_{-}}>}{\left(\omega-\omega_{e_{1_{-}} g}+i \Gamma_{e_{1_{-}}}\right)} \cdot \sum_{n_{ \pm}} \frac{<e_{1_{-}}\left|\left(\vec{\mu}+\vec{\mu}^{\prime}\right)\right| e_{n_{ \pm}}>\times<e_{n_{ \pm}}\left|\left(\vec{\mu}+\vec{\mu}^{\prime}\right)\right| g>}{\left(\omega_{2}-\omega_{e_{n_{ \pm}} g}\right)\left(\omega_{1}-\omega_{e_{n_{ \pm}}} g\right.}\right) \\
& \equiv \frac{A_{+}}{\left(\omega-\omega_{e_{1_{+}} g}+i \Gamma_{e_{1_{+}} g}\right)}+\frac{A_{-}}{\left(\omega-\omega_{e_{1_{-}} g}+i \Gamma_{e_{1_{-}} g}\right)}
\end{aligned}
$$

where we have neglected the damping factors in the "off-resonant" denominators. With $\mid g>$ and $\mid e_{n_{ \pm}}>$given by Eqs. (4) and (9), the quantities $A_{ \pm}$can be expressed in the form:

$$
\begin{aligned}
A_{ \pm} & = \pm \frac{\left(\omega_{1}-\omega_{2}\right)}{4 \hbar^{2}}\left[\frac{\left(\vec{\mu}_{01}+\vec{\mu}_{01}^{\prime}\right) \cdot\left(\Delta \vec{\mu}_{10}-\Delta \vec{\mu}_{10}^{\prime}\right) \times\left(\vec{\mu}_{10}-\vec{\mu}_{10}^{\prime}\right)}{\left(\omega_{2}-\omega_{e_{1_{\mp}} g}\right)\left(\omega_{1}-\omega_{e_{1_{\mp}} g}\right)}+\right. \\
& \left.+\sum_{n \geq 2}\left(\vec{\mu}_{01}+\vec{\mu}_{01}^{\prime}\right) \cdot\left(\frac{\left(\vec{\mu}_{1 n}+\vec{\mu}_{1 n}^{\prime}\right) \times\left(\vec{\mu}_{n 0}+\vec{\mu}_{n 0}^{\prime}\right)}{\left(\omega_{2}-\omega_{e_{n+} g}\right)\left(\omega_{1}-\omega_{e_{n+} g}\right)}+\frac{\left(\vec{\mu}_{1 n}-\vec{\mu}_{1 n}^{\prime}\right) \times\left(\vec{\mu}_{n 0}-\vec{\mu}_{n 0}^{\prime}\right)}{\left(\omega_{2}-\omega_{e_{n-} g}\right)\left(\omega_{1}-\omega_{e_{n-}}\right)}\right)\right]
\end{aligned}
$$

with $\vec{\mu}_{a b}$ and $\vec{\mu}_{a b}^{\prime}$ denoting the transition dipole moments of the two monomers between states $\mid a>$ and $\mid b>, \Delta \vec{\mu}_{10} \equiv \vec{\mu}_{11}-\vec{\mu}_{00}$, and $\Delta \vec{\mu}_{10}^{\prime} \equiv \vec{\mu}_{11}^{\prime}-\vec{\mu}_{00}^{\prime}$.

If we neglect the difference between $\omega_{e_{n_{+}} g}$ and $\omega_{e_{n_{-}} g}$ in the above expression, consider $\vec{\mu}_{0 n}$ and $\vec{\mu}_{1 n}$ to be real, and realize that $\vec{\mu}_{1 i} \cdot\left(\vec{\mu}_{i 0} \times \vec{\mu}_{10}^{\prime}\right)=\vec{\mu}_{1 i}^{\prime} \cdot\left(\vec{\mu}_{i 0}^{\prime} \times \vec{\mu}_{10}\right)$ from the $C_{2}$ symmetry of the dimer, we can simplify Eq. (12) to:

$$
A_{ \pm}= \pm \frac{\left(\omega_{1}-\omega_{2}\right)}{4 \hbar^{2}}\left[\left\{\frac{2 \Delta \vec{\mu}_{10} \cdot\left(\vec{\mu}_{10} \times \vec{\mu}_{10}^{\prime}\right)}{\left(\omega_{2}-\omega_{e_{1} g}\right)\left(\omega_{1}-\omega_{e_{1} g}\right)}+\sum_{n \geq 2} \frac{4 \vec{\mu}_{1 n} \cdot\left(\vec{\mu}_{n 0} \times \vec{\mu}_{10}^{\prime}\right)}{\left(\omega_{2}-\omega_{e_{n} g}\right)\left(\omega_{1}-\omega_{e_{n} g}\right)}\right\}\right.
$$




$$
\left.+\sum_{n \geq 2} \frac{4 \vec{\mu}_{1 n} \cdot\left(\vec{\mu}_{n 0} \times \vec{\mu}_{10}\right)}{\left(\omega_{2}-\omega_{e_{n} g}\right)\left(\omega_{1}-\omega_{e_{n} g}\right)}\right]
$$

with $\omega_{e_{n} g} \equiv \frac{\omega_{e_{n+} g}+\omega_{e_{n-}}}{2}$. Note that the last term vanishes if the monomer components of the dimer molecule are achiral. The terms in the curly brackets, however, can be nonzero even with achiral monomers as long as the dimer structure is chiral. Thus, for the case of BN, the nonlinear optical chiral response must come from the terms in the curly brackets. As a further simplification, we neglect the dispersion of the denominators in the summation in Eq. (13) and replace the transition frequencies $\omega_{e_{n} g}$ by a common effective transition frequency $\omega_{e f f}$. This approximation transforms the system into an effective three-level system. We then have, with the help of the closure relation of eigenstates,

$$
\begin{aligned}
& \sum_{n \geq 2} \frac{4 \vec{\mu}_{1 n} \cdot\left(\vec{\mu}_{n 0} \times \vec{\mu}_{10}^{\prime}\right)}{\left(\omega_{2}-\omega_{e_{n} g}\right)\left(\omega_{1}-\omega_{e_{n} g}\right)} \approx \frac{-4 \Delta \vec{\mu}_{10} \cdot\left(\vec{\mu}_{10} \times \vec{\mu}_{10}^{\prime}\right)}{\left(\omega_{2}-\omega_{e f f}\right)\left(\omega_{1}-\omega_{e f f}\right)} \\
& \sum_{n \geq 2} \frac{4 \vec{\mu}_{1 n} \cdot\left(\vec{\mu}_{n 0} \times \vec{\mu}_{10}\right)}{\left(\omega_{2}-\omega_{e_{n} g}\right)\left(\omega_{1}-\omega_{e_{n} g}\right)} \approx 0
\end{aligned}
$$

and hence,

$$
A_{ \pm}= \pm \frac{\left(\omega_{1}-\omega_{2}\right)}{4 \hbar^{2}} \Delta \vec{\mu}_{10} \cdot\left(\vec{\mu}_{10} \times \vec{\mu}_{10}^{\prime}\right)\left[\frac{2}{\left(\omega_{2}-\omega_{e_{1} g}\right)\left(\omega_{1}-\omega_{e_{1} g}\right)}-\frac{4}{\left(\omega_{2}-\omega_{e f f}\right)\left(\omega_{1}-\omega_{e f f}\right)}\right]
$$

We can now use Eqs. (10), (11) and (15) to calculate $\chi_{\text {chiral }}$ versus $\omega$ for BN and compare the result with experiment. The BN molecule (Fig. 1) is a dimer with two connected naphthalene subunits twisted in orientation by $\sim 100^{\circ}[14]$. The $\mid e_{1_{+}}>$state lies above the $\mid e_{1_{-}}>$[14]. From Table 8-3 of Ref. [15], we find $\left|\Delta \vec{\mu}_{10}\right|=0.5$ Debye and from Ref. [14], we find $\left|\vec{\mu}_{10}\right|=1.4$ Debye with $\vec{\mu}_{10}$ lying in the monomer plane and making an angle of $\theta_{1}=40^{\circ}$ with the short axis of the monomer. The direction of $\Delta \vec{\mu}_{10}$ is not known, but we assume that it lies along the long axis of the monomer, making an angle of $\theta_{0}=90^{\circ}$ with the short axis. The geometry of BN in Fig. 1 shows that:

$$
\Delta \vec{\mu}_{10} \cdot\left(\vec{\mu}_{10} \times \vec{\mu}_{10}^{\prime}\right)=\left|\Delta \vec{\mu}_{10}\right|\left|\vec{\mu}_{10}\right|^{2} \cdot \sin (\alpha) \sin \left(\theta_{1}\right) \sin \left(\theta_{1}-\theta_{0}\right)
$$

Finally, we take $\hbar \omega_{e f f}=6 \mathrm{eV}$ to roughly coincide with the position of the first strong absorption peak of BN. With $\hbar \omega_{1}$ fixed at $1.17 \mathrm{eV}$ and $\omega_{2}$ varied, the calculated $\mathrm{SF}$ spectrum of 
$\left|\chi_{\text {chiral }} / N\right|$ versus $\omega$ is displayed in Fig. 2 in comparison with the experimental spectrum [5]. In the calculation, we used $\hbar \omega_{e_{1_{+}} g}=3.90 \mathrm{eV}, \hbar \omega_{e_{1_{-}} g}=3.69 \mathrm{eV}$, and $\Gamma_{{1_{1}}_{ \pm} g}=500 \mathrm{~cm}^{-1}$. The local field factors $L(\omega), L\left(\omega_{1}\right)$, and $L\left(\omega_{2}\right)$ described in Eq. (10) were found to be 1.39, 1.32 , and 1.33 , respectively. The agreement between theoretical and experimental spectra is fairly good except for the larger theoretical values of the peak amplitudes.

The discrepancy in the absolute values of the peak amplitudes between theory and experiment is expected from the assumptions and simplifications used in the model calculation. Most importantly, we do not know the direction of $\Delta \vec{\mu}_{10}$. If the angle $\theta_{0}$ is closer to $40^{\circ}$ than $90^{\circ}$, the absolute values of $A_{ \pm}$will be much reduced, as can be seen from Eqs. (15) and (16). An $a b$ initio calculations on the monomer part of BN should be able to yield the direction of $\Delta \vec{\mu}_{10}$ and then allow us to predict better values for $A_{ \pm}$.

The value of $\theta_{0}$ could be deduced from a doubly resonant SFG experiment with $\omega_{1} \approx \omega_{e_{1} g}$ and $\omega \approx \omega_{e_{1_{+}} g}$. In this case, the terms with $n \geq 2$ in the expression of $\chi_{\text {chiral }}$ can be neglected, and the system becomes more truly a three-level system. The value of $\theta_{0}$ can be obtained by fitting an SFG spectrum near double resonance. More generally, the two-dimensional doubly resonant SFG spectrum can provide a more rigorous check of the coupled-oscillator model discussed here.

The twist angle $\alpha$ between the two monomers in $\mathrm{BN}$ is obviously responsible for the molecular chirality of BN. The two enantiomers, S-BN and R-BN, have $\alpha=+100^{\circ}$ and $-100^{\circ}$, respectively, yielding opposite signs for $\chi_{\text {chiral }}$. Equation (15) as an approximation also shows that for nonvanishing $\chi_{\text {chiral }}$ the three vectors $\Delta \vec{\mu}_{10}, \vec{\mu}_{10}$ and $\vec{\mu}_{10}^{\prime}$ must not be in the same plane: $\vec{\mu}_{10}$ and $\vec{\mu}_{10}^{\prime}$ must not be along the short axis of the monomers and $\Delta \vec{\mu}_{10}$ must be tilted away from $\vec{\mu}_{10}$.

It is interesting to know how linear and nonlinear optical activity measurements provide similar or different chiral structural information about the chiral molecules. The linear optical activity arises from the difference of refractive indices, $n_{L}$ and $n_{R}$, of the chiral medium for left and right circularly polarized light, respectively, in a chiral medium. For a chiral liquid we have [9]: 


$$
n_{R}-n_{L}=\frac{2}{3} c \mu_{0} N L(\omega) \cdot \frac{2}{\hbar} \sum_{n \neq g} \frac{\omega \cdot R_{n g}}{\left(\omega-\omega_{n g}+i \Gamma_{n g}\right)\left(\omega+\omega_{n g}-i \Gamma_{n g}\right)}
$$

where $R_{n g} \equiv \operatorname{Im}\left(\vec{\mu}_{g n} \cdot \vec{m}_{n g}\right), \omega$ is the light frequency, $\vec{\mu}$ and $\vec{m}$ are electric and magnetic dipole operators, respectively, and we have assumed that only the ground state is populated.

Using the coupled-oscillator model, we can find for a dimer molecule composed of two achiral monomers, [9]:

$$
R_{e_{n_{ \pm}} g}= \pm \frac{1}{4} \omega_{n g} \vec{R} \cdot\left(\vec{\mu}_{n 0}^{\prime} \times \vec{\mu}_{n 0}\right)
$$

where $\vec{R}$ is the vector connecting the centers of the monomers. With $\omega \sim \omega_{e_{1_{ \pm}} g}$, we can write:

$$
\frac{n_{R}-n_{L}}{N} \approx \frac{B_{+}}{\omega-\omega_{e_{1_{+}} g}+i \Gamma_{e_{1_{+}} g}}+\frac{B_{-}}{\omega-\omega_{e_{1_{-}} g}+i \Gamma_{e_{1} g}}
$$

with

$$
B_{ \pm}= \pm \frac{1}{3 \hbar} c \mu_{0} \frac{\omega \omega_{e_{1} g}}{\omega+\omega_{e_{1} g}} \vec{R} \cdot\left(\vec{\mu}_{10}^{\prime} \times \vec{\mu}_{10}\right)
$$

We can now compare the chiral structural information contained in linear and nonlinear optical activities as represented by $B_{ \pm}$in Eq. (20) and $A_{ \pm}$in Eq. (13), respectively. In both cases, if the two achiral monomers comprising the molecule are not coupled so that $\omega_{e_{1_{+} g}}=\omega_{e_{1_{-}} g}$, then optical activities must vanish. The chiral response in linear optical activity comes from the vector product $\vec{R} \cdot\left(\vec{\mu}_{10}^{\prime} \times \vec{\mu}_{10}\right)$ (for BN molecules, $\vec{R} \cdot\left(\vec{\mu}_{10}^{\prime} \times \vec{\mu}_{10}\right)=$ $|\vec{R}|\left(\left|\vec{\mu}_{10}\right|^{2} \sin (\alpha) \sin \left(\theta_{1}\right)\right)$ while that in nonlinear optical activity comes from $\Delta \vec{\mu}_{10} \cdot\left(\vec{\mu}_{10} \times \vec{\mu}_{10}^{\prime}\right)$ and $\vec{\mu}_{1 n} \cdot\left(\vec{\mu}_{n 0} \times \vec{\mu}_{10}^{\prime}\right)$. In the linear case only the transition between monomer states $\mid 0>$ and $\mid 1>$ is involved in $B_{ \pm}$. The magnitude of $B_{ \pm}$depends on $|\vec{R}|$ and the geometric angle between $\vec{R}$ and $\vec{\mu}_{10}^{\prime} \times \vec{\mu}_{10}$. The former means that the strength of optical activity associated with each resonance actually increases with separation of the two monomers in the molecules. It originates from the fact that the linear optical activity response is connected to a magnetic dipole matrix element and therefore is nonlocal. This is not the case for nonlinear optical activity since $A_{ \pm}$is independent of $\vec{R}$. Instead, $\Delta \vec{\mu}_{10}$ plays the role of $\vec{R}$ if contributions from 
higher excited states can be neglected. More generally, higher off-resonant excited states also contribute to the resonant nonlinear optical activity through $\vec{\mu}_{1 n} \cdot\left(\vec{\mu}_{n 0} \times \vec{\mu}_{10}^{\prime}\right)$, which is a vector product of three transition dipole moments. The nonlinear optical activity strength is connected to vector products of electric-dipole moments and therefore is local in response (independent of $\vec{R}$ ). Thus clearly linear and nonlinear optical activities provide very different information about the chiral structure or property of a dimer molecule.

We note that resonant linear optical activity can be used to probe selectively how each electronic transition contributes to electronic chirality of a chiral dimer through $\vec{R} \cdot\left(\vec{\mu}_{10}^{\prime} \times \vec{\mu}_{10}\right)$. On the other hand, while singly resonant SFG is less selective in probing chirality, doubly resonant SFG can selectively probe the contribution of $\vec{\mu}_{0 n} \cdot\left(\vec{\mu}_{n n^{\prime}} \times \vec{\mu}_{n^{\prime} 0}^{\prime}\right)$ to the chiral response. The idea can be extended to double resonance involving one electronic transition and one vibrational transition. Then, more chiral structural information about the molecule can be anticipated from the result.

In summary, we have shown in this paper that a simple coupled-oscillator model can be used to describe SF nonlinear optical activity of a dimer-like chiral molecule. The result of calculation for chiral BN solution agrees well with experiment except for uncertainty in the absolute strength of chirality. The same model should also describe optically active SHG from a surface monolayer if the orientational distribution of $\mathrm{BN}$ is properly taken into account. Nonlinear optical activity probes different aspects of the chiral structure of the dimer-like molecule than linear optical activity. We anticipate that doubly resonant SFG can yield highly selective, hitherto unexplored, information about chirality of such molecules.

\section{ACKNOWLEDGMENTS}

This work was supported by the Director, Office of Science, Office of Basic Energy Sciences, Division of Material Sciences and Engineering of the U.S. Department of Energy and the Laboratory Director Research and Development Program of Lawrence Berkeley National Laboratory under Contract No. DE-AC03-76SF00098. C.F. would like to thank the Miller 
Institute and the Lawrence Berkeley National Laboratory of the University of California at Berkeley for financial support during his stay at Berkeley. We acknowledge valuable discussions with Professor Robert A. Harris. 


\section{REFERENCES}

[1] T. Petralli-Mallow, T. M. Wong, J. D. Byers, H. I. Yee, and J. M. Hicks, J. Phys. Chem. 97, 1383 (1993). J. D. Byers, H. I. Yee, T. Petralli-Mallow, and J. M. Hicks, Phys. Rev. B 49, 14643 (1994).

[2] Jeffery J. Maki, Martii Kauranen, and André Persoons, Phys. Rev. B 51, 1425 (1995). Martii Kauranen, Thierry Verbuest and André Persoons, J. Mod. Opt. 45, 403 (1998).

[3] M. A. Belkin, T. A. Kulakov, K. H. Ernst, L. Yan, and Y. R. Shen, Phys. Rev. Lett. 85, $4474(2000)$.

[4] M. A. Belkin, S.-H. Han, X. Wei, and Y. R. Shen, Phys. Rev. Lett. 231, article \#11300 (2001).

[5] The experimental spectrum of $\left|\chi_{\text {chiral }} / N\right|$ was deduced from a recent, more carefully measured SFG spectrum from a $0.7 \mathrm{M}$ solution of BN in tetrahydrofuran using a setup similar to that of Ref. [4]. Unlike Ref. [4], we have also included the dispersion of the coherence length in calculating $\left|\chi_{\text {chiral }} / N\right|$.

[6] F. Hache, H. Mesnil, and M. C. Schanne-Klein, J. Phys. Chem. 115, 6707 (2001).

[7] J. M. Hicks, T. Petralli-Mallow and J. D. Byers, Faraday Discuss 99, 341 (1994); J. D. Byers and J. M. Hicks, Chem. Phys. Lett. 231, 216 (1994).

[8] P. Fischer, D. S. Wiersma, R. Righini, B. Champagne, A. D. Buckingham , Phys. Rev. Lett. 85, 4253 (2000).

[9] See, for example, L. D. Barron, Molecular Light Scattering and Optical Activity (Cambridge University Press,Cambridge, UK, 1982), Ch. 5.

[10] W. Kuhn, Trans. Farafay Soc. 26, 293 (1930).

[11] Y. R. Shen, in Proceedings of the International School of Physics "Enrico Fermi", Course CXX, Frontiers in Laser Spectroscopy, edited by Hänsch, T. W. and Inguscio, M. (North 
Holland, Amsterdam, 1994), p.139.

[12] Y. R Shen, The Principles of Nonlinear Optics (J. Wiley, New York, 1984), Ch. 2.

[13] J. A. Giordmaine, Phys. Rev. 138, A1599 (1965).

[14] I. Hanazaki and H. Akimoto, J. Am. Chem. Soc. 94, 4102 (1972).

[15] N. Mataga and T. Kubota, Molecular Interactions and Electronic Spectra (Marcel Dekker, New York, 1970). 


\section{FIGURES}
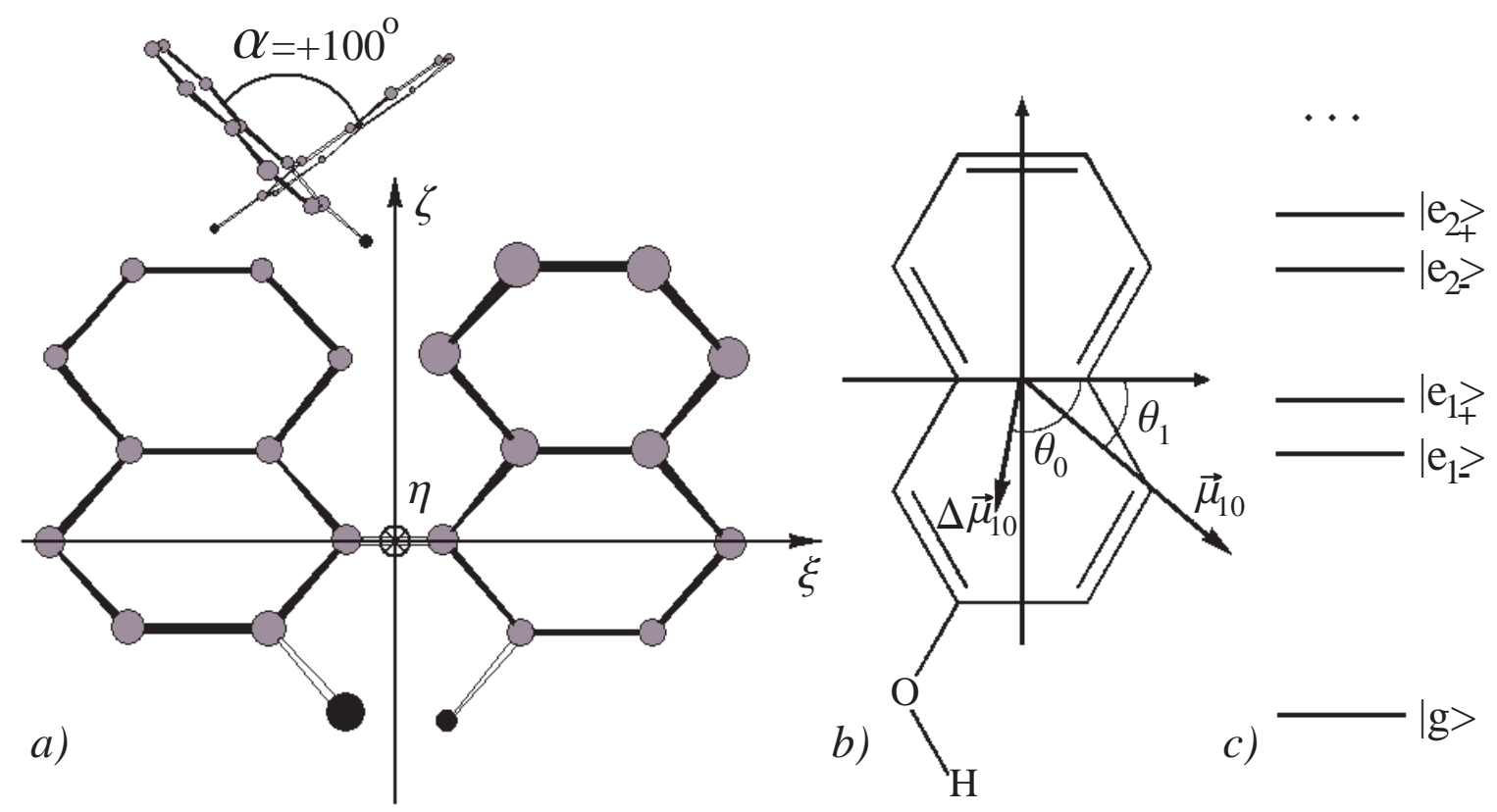

FIG. 1. (a) Structure of an S-BN molecule that is composed of a pair of monomers whose structure is described in (b). The energy level diagram of $\mathrm{BN}$ is sketched in (c). 


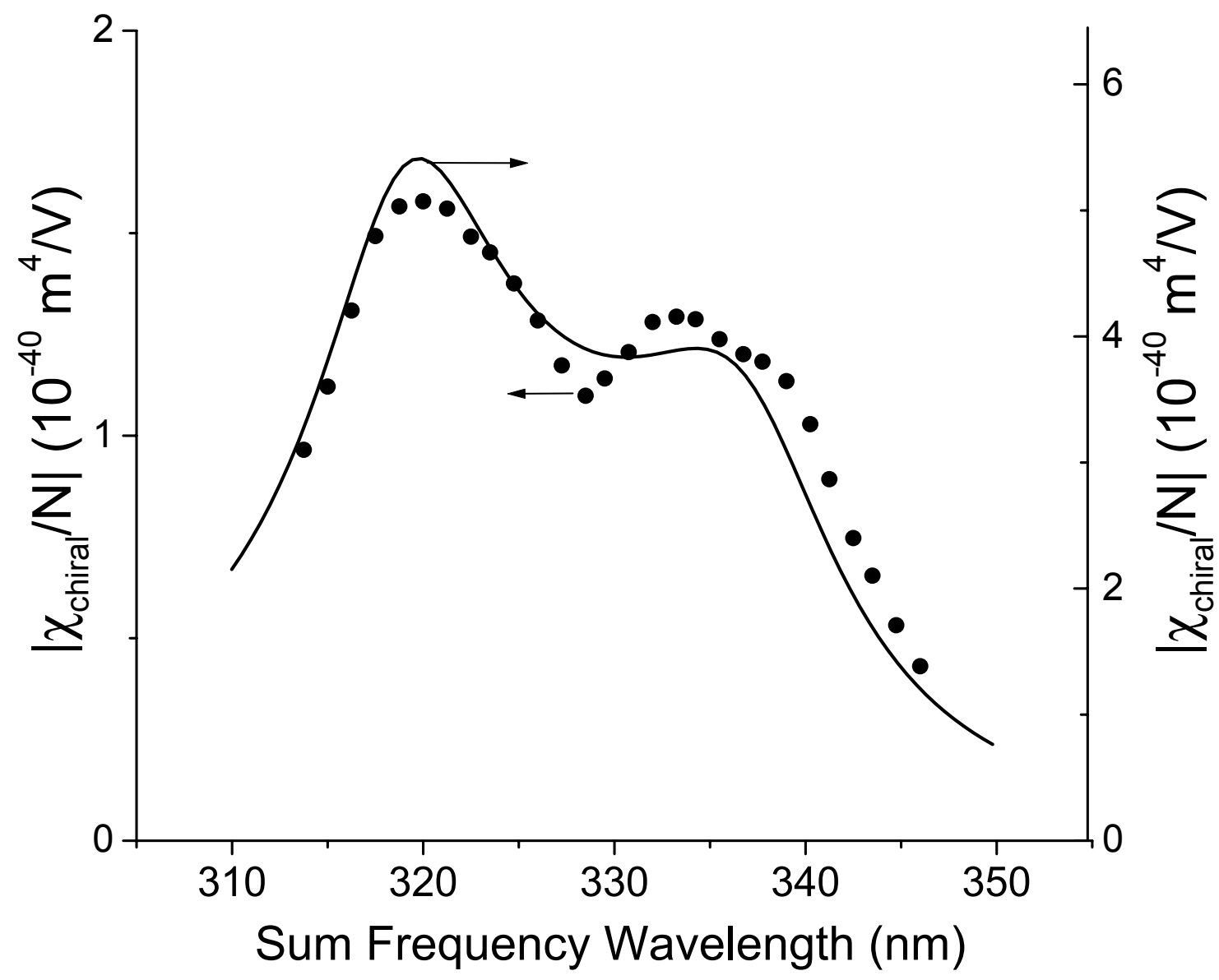

FIG. 2. Experimental result (dots) and model prediction (solid line) of $\left|\chi_{\text {chiral }} / N\right|$ versus $\omega$ for a $0.7 \mathrm{M}$ solution of $\mathrm{BN}$ in tetrahydrofuran. 